\title{
Práticas integrativas e complementares segundo os secretários de saúde: Estudo
}

\section{qualitativo}

\author{
Integrative and complementary practices according to health secretaries: Qualitative study \\ Prácticas integradoras y complementarias según los secretarios de salud: Un estudio cualitativo
}

Recebido: 10/04/2021 | Revisado: 17/04/2021 | Aceito: 22/04/2021 | Publicado: 08/05/2021

Larissa de Oliveira Vieira

ORCID: https://orcid.org/0000-0002-5230-7181

Universidade Estadual do Sudoeste da Bahia, Brasil

E-mail: larissaovieira@ hotmail.com

Ismar Eduardo Martins Filho

ORCID: https://orcid.org/0000-0002-1013-6951

Universidade Estadual do Sudoeste da Bahia, Brasil

E-mail: iefilho@uesb.edu.br

Edméia Campos Meira

ORCID: https://orcid.org/0000-0002-8328-6918

Universidade Estadual do Sudoeste da Bahia, Brasil E-mail: edmeiameira@yahoo.com.br

\begin{abstract}
Resumo
As Práticas Integrativas e Complementares (PIC) são recursos em saúde que possibilitam o cuidado de acordo os princípios da integralidade, e estão inseridos em um modelo assistencial que extrapola a visão biomédica. O objetivo deste artigo foi compreender a percepção de secretários municipais de saúde sobre as PIC, assim como identificar quais PIC eram conhecidas por eles. Trata-se de uma pesquisa qualitativa e exploratória, realizada com 22 secretários de saúde em municípios da Bahia. A coleta de dados aconteceu entre os meses de maio e novembro de 2018 nos municípios e nas reuniões da Comissão Intergestores Regional. As entrevistas foram coletadas por meio de roteiro semiestruturado, gravadas, transcritas e analisadas através da análise de conteúdo temática. $\mathrm{O}$ estudo apontou que todos os secretários conhecem algumas PIC e também já as utilizaram enquanto recurso terapêutico, obtendo resultados positivos; identificou ainda que apenas 7 secretários conhecem a Política Nacional de Práticas Integrativas e Complementares (PNPIC) e os municípios não têm lei que regulamente tais PIC; obteve-se também que apenas 5 secretários estudaram as PIC na formação acadêmica. É importante que os secretários conheçam as PIC e seus benefícios como recursos potentes para a saúde, mas não conhecer a PNPIC pode dificultar a operacionalização da implantação das PIC nas regiões de saúde em que eles são gestores. Faz-se necessário a articulação da população, equipes de saúde e gestores de saúde na discussão e elaboração de projetos e programas que fortaleçam as PIC enquanto recurso de saúde potente para a atenção integral em saúde.
\end{abstract}

Palavras-chave: Práticas integrativas e complementares; Gestão em saúde; Integralidade em saúde; Sistema único de saúde.

\begin{abstract}
The Integrative and Complementary Practices (PIC) are health resources that enable care according to the principles of integrality, and are inserted into a care model that goes beyond the biomedical vision. The objective of this article was to understand the perception of municipal health secretaries about PICs, as well as to identify which PICs were known by them. This is a qualitative and exploratory research, carried out with 22 health secretaries in municipalities of Bahia. Data collection took place between the months of May and November 2018 in the municipalities and in the meetings of the Regional InterManagerial Commission. The interviews were collected through a semi-structured script, recorded, transcribed, and analyzed through thematic content analysis. The study pointed out that all the secretaries know some PIC and have also used them as a therapeutic resource, obtaining positive results; it also identified that only 7 secretaries know the National Policy of Integrative and Complementary Practices (PNPIC) and the municipalities have no law that regulates such PIC; it was also obtained that only 5 secretaries studied PIC in their academic training. It is important that the secretaries know about the PICs and their benefits as powerful resources for health, but not knowing the PNPIC can hinder the operationalization of the implementation of PICs in the health regions where they are managers. It is necessary to articulate the population, health teams, and health managers in the discussion and development of projects and programs that strengthen the PICs as a powerful health resource for comprehensive health care.
\end{abstract}

Keywords: Integrative and complementary practices; Health management; Integrality in health; Unified health system. 


\begin{abstract}
Resumen
Las Prácticas Integradoras y Complementarias (PIC) son recursos sanitarios que permiten la atención según los principios de la integralidad, y se insertan en un modelo de atención que supera la visión biomédica. El objetivo de este artículo era conocer la percepción de los secretarios municipales de salud sobre los CFP, así como identificar qué CFP eran conocidos por ellos. Se trata de una investigación cualitativa y exploratoria, realizada con 22 secretarios de salud de municipios de Bahía. La recogida de datos se realizó entre mayo y noviembre de 2018 en los municipios y en las reuniones del Comité Interinstitucional Regional. Las entrevistas se recogieron mediante un guión semiestructurado, se grabaron, se transcribieron y se analizaron mediante un análisis de contenido temático. El estudio señaló que todas las secretarias conocen alguna PIC y también las han utilizado como recurso terapéutico, obteniendo resultados positivos; también se identificó que sólo 7 secretarias conocen la Política Nacional de Prácticas Integrativas y Complementarias (PNPIC) y los municipios no tienen ninguna ley que regule dichas PIC; también se obtuvo que sólo 5 secretarias estudiaron PIC en su formación académica. Es importante que los secretarios conozcan el PIC y sus beneficios como poderosos recursos para la salud, pero el desconocimiento del PNPIC puede dificultar la operatividad de la implementación del PIC en las regiones sanitarias donde son gestores. Es necesaria la coordinación de la población, los equipos de salud y los gestores sanitarios en la discusión y desarrollo de proyectos y programas que fortalezcan el CFP como un poderoso recurso sanitario para la atención integral de la salud.

Palabras clave: Prácticas integradoras y complementarias; Gestión de la salud; Integridad en la salud; Sistema de salud unificado.
\end{abstract}

\title{
1. Introdução
}

As Práticas Integrativas e Complementares (PICS) são recursos em saúde que acompanham a mudança do paradigma do modelo de atenção à saúde e defendem o cuidado integral, de modo a compreender as pessoas na tríade corpo-mente-alma (Fischborn et al., 2016). Estas práticas foram regulamentadas por meio de diversas portarias e são compostas por: Acupuntura, Homeopatia, Fitoterapia e o Termalismo social/crenoterapia, Arteterapia, Ayurveda, Biodança, Dança Circular, Meditação, Musicoterapia, Naturopatia, Osteopatia, Quiropraxia, Reflexoterapia, Reiki, Shantala, Terapia Comunitária Integrativa e Yoga (Brasil, 2015).

A partir da publicação da Política Nacional de Práticas Integrativas e Complementares (PNPIC) há 12 anos, houve maior visibilidade e ampliação da utilização das práticas nos serviços de saúde (Brasil, 2015). Entre os objetivos da Política está o de estimular as ações referentes ao controle/participação social, promovendo o envolvimento responsável e continuado dos usuários, gestores e trabalhadores nas diferentes instâncias de efetivação das políticas de saúde (Brasil, 2015). Algumas dificuldades para a consolidação das PIC são elencadas a exemplo da falta de investimento financeiro, poucos estudos na área além da necessidade de maior conhecimento entre gestores, profissionais e usuários a respeito das PIC e suas potencialidades (Contatore et al., 2015).

A Organização Mundial da Saúde (OMS) ao realizar um levantamento com diversos países em relação às dificuldades enfrentadas para a regulamentação das práticas tradicionais e complementares, identificou entre as principiais queixas a insuficiência de dados de pesquisas e a falta de apoio financeiro para estas pesquisas, além da falta de conhecimento especializado entre as autoridades de saúde e órgãos nacionais de controle (Who, 2013).

Diante deste cenário, esta pesquisa teve o objetivo de compreender o conhecimento de secretários municipais de saúde sobre as PIC e aspectos pertinentes a esta temática, assim como identificar quais PIC são conhecidas por eles.

\section{Metodologia}

Trata-se de um estudo exploratório e descritivo, de abordagem qualitativa. A pesquisa foi realizada com secretários de saúde do Núcleo Regional de Saúde-Sul (NRS-Sul), onde atualmente ocorrem as reuniões da Comissão Intergestores Regional (CIR). O NRS-Sul é composto por 26 municípios e está sob a coordenação do município de Jequié, município que fica situado na região sudoeste do Estado da Bahia, distando $364 \mathrm{~km}$ da capital, Salvador. Os 26 municípios do NRS-Sul têm uma população total de 491.709 habitantes, e o percentual da população do NRS-Sul em relação ao estado da Bahia é de $3,31 \%$, uma vez que a Bahia tem uma população estimada de 14.812.617 (IBGE, 2018). 
Foram convidados a participar do estudo os 26 secretários de saúde que compõem a região de saúde de Jequié, e apenas 22 secretários participaram da pesquisa, por motivo de saúde ou de viagens para representar as secretarias de saúde os gestores não foram encontrados nas visitas, além de algumas reuniões terem sido desmarcadas, o que impossibilitou o contato com os 4 secretários de saúde. A amostra da pesquisa se deu por exaustão, em que são incluídos todos os indivíduos disponíveis, pensando na representatividade desses participantes e na qualidade das informações obtidas deles (Fontanella, Ricas \& Turato, 2008).

Como instrumento de coleta de dados foi utilizado um roteiro de entrevista semiestruturado elaborado pelos pesquisadores do estudo e compostos de questões sociodemográficas e específicas acerca do tema da PNPIC, que contemplavam questionamentos pertinentes aos objetivos do estudo. A coleta ocorreu no período entre os meses de maio e novembro de 2018. Os secretários foram entrevistados em um único momento e as entrevistas realizadas foram feitas individualmente. Das entrevistas, 15 ocorreram antes do início das reuniões da CIR em Jequié, tendo uma duração média de 15 minutos cada. As outras 7 entrevistas ocorreram depois de visitas realizadas nos municípios, nos espaços das Secretarias de Saúde e tiveram uma duração média de 25 minutos cada. Os entrevistados foram identificados pela letra "E" (entrevistado) seguido pela numeração correspondente à ordem das entrevistas.

Os dados primários provenientes das entrevistas foram analisados de acordo com a análise de conteúdo temática, que possibilitou a codificação, classificação e categorização dos dados após uma leitura exaustiva do material, considerando a fundamentação teórica, o problema e os objetivos do estudo (Bardin, 2011). Da análise das transcrições das entrevistas obtidas emergiram categorias temáticas: 1) conhecimento da PNPIC e das PIC; 2) uso e benefícios terapêuticos das PIC; 3) a temática das PIC na formação acadêmica dos secretários de saúde.

A presente pesquisa é resultado de dissertação de mestrado e foi autorizada pelo comitê de ética em pesquisa com seres humanos, CAAE n. 79083817.0.0000.0055, pelo parecer n. 2.627.055 /2018. A aceitação dos participantes para participar da pesquisa se deu de forma voluntária, mediante assinatura do Termo de Consentimento Livre e Esclarecido.

\section{Resultados}

Dos participantes, $72,7 \%(\mathrm{n}=22)$ eram mulheres e em relação à faixa etária, $12(54,5 \%)$ tinham entre 30 a 40 anos, 7 $(31,8 \%)$ tinham a faixa etária de 41 a 50 anos, $2(9,0 \%)$ tinham idade entre 51 e 60 anos e apenas $1(0,45 \%)$ participante tinha idade entre 18 e 29 anos. Do total de 22 participantes do estudo: 12 (54,5\%) tinham nível superior em enfermagem, 5 (22,7\%) nível superior em administração, e as categorias profissionais serviço social, farmácia, bacharel em direito tiveram cada uma 1 secretário com nível superior. Dos outros 2 secretários, 1 possuía nível médio e 1 nível superior incompleto, cursando história. Nenhum contém titulação a nível de mestrado ou doutorado. Dentre os participantes, $15(68,1 \%)$ atuam no cargo de 1 ano a 1 ano e 8 meses e 6 (36,3\%) estão no cargo por 11 meses ou menos, apenas 1 secretário possui mais de 2 anos no cargo.

\section{1) Conhecimento da PNPIC e das PIC}

No que se refere ao conhecimento da PNPIC, dos 22 entrevistados, apenas 7 responderam que já tinham ouvido falar da Política. Apenas dois secretários se manifestaram quanto à temática, os outros apenas responderam sim ou não.

Não. A gente sempre recebe alguma nota técnica falando sobre, mas a Política não (E 6). Já li, mas nada aprofundado (E 18).

Já ao serem questionados sobre o termo PIC, dos 22 entrevistados, 16 responderam de maneira positiva que conheciam as PIC, os outros secretários demonstraram desconhecer o termo. 
Sim. Mas com termos diferentes (E 4).

Já ouvi, sei pouco, entendo que é complementar as atividades da unidade. Exemplo o paciente que acredita que a doença só melhora com fitoterápico (E 14).

Sim. Tenho conhecimento vago. Entendo que seja algo que venha integrar e complementar as ações normais de saúde que a gente aplica no dia a dia (E 19).

Ao mostrar as 29 PIC dispostas em um quadro, por ano de suas regulamentações, obteve-se que as PIC que os secretários mais conheciam eram as plantas medicinais/fitoterapia, acupuntura e homeopatia, implantadas incialmente com a publicação da PNPIC em 2006, além da yoga, meditação, musicoterapia, implantadas em 2017, enquanto as PIC mais conhecidas a partir da portaria de 2018 foram a cromoterapia e aromaterapia

Destaca-se que as Plantas Medicinais e Fitoterapia (Chás, ervas) foram conhecidas por 18 secretários, Yoga por 15, Medicina Tradicional Chinesa/ Acupuntura, Meditação e Homeopatia por 14, Musicoterapia por 10, Shantala, Terapia Comunitária Integrativa e Arteterapia por 7, Naturopatia, Cromoterapia e Medicina Tradicional Chinesa/auriculoterapia por 6, Biodança e Aromaterapia por 5, Dança Circular, Terapia de Florais e Ozonioterapia por 4, Reflexoterapia, Quiropraxia e Hipnoterapia por 3, Constelação familiar, Reiki e Termalismo Social/ Crenoterapia por 2, Medicina Antroposófica, Osteopatia, Imposição de mãos e Geoterapia por 1. Nenhum secretário conhecia as práticas Ayurveda, Apiterapia e Bioenergética.

\section{2) Uso e benefícios terapêuticos das PIC}

Quando questionados se já tinham usado alguma PIC como recurso de saúde para algum tipo de adoecimento, dos 22 secretários entrevistados, 12 responderam positivamente. No total, as plantas medicinais foram utilizadas por 8 , a auriculoterapia e a homeopatia por 4, a acupuntura por 3, a yoga por 2, e as PIC constelação familiar, cromoterapia, musicoterapia, quiropraxia, reiki e terapia de florais foram citadas cada uma por apenas 1 secretário. As práticas corporais/dança também apareceram entre as respostas, sendo apontada por 1 secretário.

Alguns se manifestaram acerca do efeito do uso da auriculoterapia:

Auriculoterapia é maravilhoso, relaxa mesmo. Melhorou a minha ansiedade e diminuiu algumas dores, porque tem pontos na orelha que correspondem às dores no corpo. Eu tinha uma dor plantar muito intensa, e aí a dor parou, então realmente resolve (E 18).

Auriculoterapia é muito válido. Você coloca os pontos na orelha, você sente um pouco de dor naquele local, mas o corpo parece que cessa a dor. É incrivel (E 3).

Auriculoterapia eu peguei em pontos de estresse, para desestressar, pra mim foi bastante eficiente (E 9).

Houve ainda algumas considerações dos benefícios da acupuntura e sua eficácia:

Usei Acupuntura para hérnia de disco, aliviou bastante (E 22).

Eu vi resultado com familiares, que tem sequela de AVC e 2 hérnias de disco. Teve um efeito fantástico (E 3).

Outra PIC mencionada foi a quiropraxia: 
A quiropraxia funciona, sem dúvida! A gente já vê os resultados, principalmente para quem tem problemas de coluna (E 19).

A musicoterapia foi mencionada por um secretário enquanto recurso de saúde eficaz para o relaxamento.

Musicoterapia em trabalhos de partos é interessante porque as mulheres relaxam durante o processo do parto (E 18).

Apenas 2 secretários falaram do uso da homeopatia, e mencionaram não acreditar na eficácia da PIC.

A homeopatia eu fiz com florais, não vi muito resultado, na época eu tava na faculdade, você espera a potência da medicação e você não vê. A homeopatia é ao longo prazo (E 9).

Homeopatia eu não acredito muito não, porque é a diluição da medicação em várias proporções, mas parentes já fizeram uso de homeopatia e por incrível que pareça funcionou, a minha crença não era a mesma crença da mãe dele (E 18).

\section{3) A temática das PIC na formação acadêmica dos secretários de saúde}

Ainda referente aos questionamentos das PIC, apenas 5 dos secretários entrevistados disseram que na formação acadêmica tiveram contato com alguma das práticas, sendo que dos 22 entrevistados, 13 eram de diversas categorias profissionais da área da saúde.

Não, me formei em 2008 e não ouvi falar (E 6).

Não. Fala que existe, mas não se aprofunda, não tem aulas específicas. Agora pode ser que tenha. Me formei em 2006 e este assunto não foi abordado na academia (E 18).

Não me recordo. Nunca dá ênfase para estas questões (E 3).

Os que estudaram as PIC na formação acadêmica mencionaram:

Homeopatia, Plantas medicinais, acupuntura (E 4).

Formei em 2007. Ouvia falar em homeopatia, porque a gente estuda medicação, mas nada aprofundado (E 9).

Homeopatia, Plantas medicinais, acupuntura (E 12).

Plantas medicinais. E quando eu comecei a trabalhar eu implantei no fundo da unidade de saúde. Teve a adesão deles principalmente a população idosa (E 19).

Apenas as Plantas medicinais (E 20).

Das PIC, as plantas medicinais foram estudadas por 4 deles, já a homeopatia por 3 e a acupuntura por 2.

\section{Discussão}

O estudo abordou a compreensão de secretários municipais de saúde sobre as PIC e aspectos que abarcam esta temática. Em relação ao perfil dos participantes, identificou-se que a grande maioria tinha nível superior na área da saúde e 
notou-se também o aspecto da rotatividade dos cargos, uma vez que apenas 1 secretário estava há 24 meses na função, sendo que a gestão do último mandato de prefeito iniciou-se há 24 meses. A alta rotatividade de gestores municipais de saúde facilita um maior controle a respeito da agenda e maior poder de argumentação de gestores mais experientes, o que influencia nas votações das pautas e propostas de ações na região de saúde, além de dificultar a apropriação de conhecimentos necessários para a tomada de decisão pelos gestores com menos tempo na função (Santos \& Giovanella, 2014).

No que diz respeito ao conhecimento da temática da PNPIC, dos 22 entrevistados, apenas 7 responderam que conheciam a Política. Uma pesquisa realizada com secretários de saúde do Estado de São Paulo acerca do conhecimento da PNPIC identificou que dos 33 secretários apenas 11 (33\%) conheciam a política (Galhardi, Barros \& Leite-Mor, 2015). Este desconhecimento da PNPIC também foi observado com profissionais de uma Unidade Básica de Saúde (UBS), em que dos 70 profissionais da UBS apenas $14,5 \%$ dos profissionais afirmaram conhecer a PNPIC, enquanto 29,0\% apenas ouviram falar da política e 56,5\% desconhecem a mesma (Carvalho \& Nóbrega, 2017).

O desconhecimento da PNPIC pelos secretários de saúde pode dificultar o diálogo para a implantação das PIC nos diversos serviços da Rede de Atenção à Saúde. Outro ponto a ser destacado é que a falta de apoio institucional não contribui para a mudança de um modelo promotor da saúde, desta maneira, reforça o paradigma vigente do modelo de atenção biomédico. Os profissionais ao não conhecerem a Política e suas portarias tem a possibilidade de praticar erros na execução das PIC, o que pode provocar danos e riscos à saúde dos usuários.

Um dos grandes desafios do SUS, de acordo Fertonani, Pires, Biff, e Scherer (2015) é a superação da hegemonia do modelo biomédico, centrado na doença, para um modelo assistencial orientado para a integralidade e a ampliação do conceito de saúde. As perspectivas teóricas e políticas de implementação de um novo modelo assistencial, no Brasil, necessitam ser assimilados no cotidiano dos serviços de saúde, pelos profissionais e equipes de saúde, assim como pelos usuários e suas instâncias de controle social e ainda pelos gestores da saúde (Fertonani et al., 2015).

Em nenhum dos 22 municípios da pesquisa há Política Municipal de PIC aprovada. Sobre as políticas municipais de PIC, destaca-se a institucionalização da Política Municipal de Práticas Integrativas e Complementares no município de Vitória/ES em 2013 e a oferta de PIC de maneira exitosa nos serviços de saúde (Sacramento, 2017). Neste processo houve a ação conjunta de gestores, conselheiros de saúde e técnicos da Atenção Básica (AB), sendo necessária afirmar a importância de superar os desafios para operacionalização desta Política a partir do envolvimento de atores sociais e institucionais (Sacramento, 2017).

Já em Recife, a política municipal de PIC foi desenvolvida por líderes ou instâncias superiores, destacando-se o Conselho Municipal de Saúde, os gestores e as entidades médicas. A população, os usuários e profissionais de saúde não tiveram a participação efetiva na elaboração da política, sendo importante destacar que a participação de poucos atores na construção de uma política de PIC dificulta sua consolidação e amplia a distância entre a formulação e implementação, prejudicando o alcance dos resultados esperados (Santos, Sousa, Gurgel, Bezerra \& Barros, 2014).

Quando os entrevistados foram questionados se conheciam o termo PIC, a maioria deles conhecia. Um estudo ao investigar os conhecimentos de profissionais de UBS verificou que durante a entrevista, ao substituir a nomenclatura das PIC para Terapias Alternativas, $60 \%$ relataram usar ou já ter usado principalmente por seus familiares alguma PIC (Ischkanian \& Pelicioni, 2012).

A terminologia PIC foi pensada pelo MS pois engloba tanto as racionalidades médicas vitalistas quanto as práticas terapêuticas ditas integrativas e complementares em saúde (Nascimento, Barros, Nogueira, \& Luz, M.T, 2013). Anteriormente era conhecida por Medicina Alternativa e Complementar, oferecendo grande polissemia, designando qualquer forma de cura que não seja propriamente biomédica (Luz, 2005). Mas, apesar de existirem várias definições para as práticas em saúde que não se limitam à visão puramente biomédica, é importante destacar que todas estas nomenclaturas pretendem aproximar 
abordagens convencionais e complementares de forma coordenada e que valorizam os conceitos mais amplos de promoção da saúde e prevenção de doenças (Melchart, 2018).

Não foram encontrados estudos com secretários de saúde a respeito das PIC conhecidas por este público. Uma pesquisa realizada por Carvalho e Nóbrega (2017) com profissionais de uma UBS identificou que as PIC mais conhecidas por eles foram as PIC mais relatadas pelos gestores deste estudo, incluindo a Medicina Tradicional Chinesa/Acupuntura, as Plantas Medicinais/ Fitoterapia e Homeopatia, Meditação, Musicoterapia. Por fim, as PIC que os 2 grupos menos conhecem foram a Crenoterapia/Termalismo 2,9\% (profissionais) e 9\% (secretários) e Medicina Antroposófica por 12,9\% (profissionais) e 4\% (secretários), apesar das 2 PIC terem sido implantadas em 2006.

Observa-se que na rede de atenção a saúde do SUS, há uma variedade de categorias profissionais, de modo a contemplar o conhecimento das diversas PIC. No estudo citado acima, dentre os 70 profissionais havia Assistente Social, Farmacêutico e enfermeiros (14\% dos entrevistados, a $2^{\circ}$ maior categoria, atrás dos agentes comunitários de saúde com $18 \%$ ). Já os secretários desta pesquisa, apesar do menor número, são representados por semelhantes categorias profissionais, assistente social, farmacêutico e enfermeiros (54\%).

Pertinente ao uso e benefícios terapêuticos das PIC, vários secretários relataram o uso de tais recursos em saúde. Quanto à eficácia da auriculoterapia, os que se manifestaram mencionaram que a PIC proporciona um relaxamento, melhora em dores do corpo e para desestressar. Diante das falas dos secretários, identificou-se que a Auriculoterapia foi utilizada para diversas situações de adoecimento, a exemplo da ansiedade e estresse. Estudos comprovaram que a auriculoterapia foi eficaz na diminuição de níveis de estresse e alívio da lombalgia (Albear de la Torre, Cedeño, Fajardo \& Riveaux, 2016), redução de ansiedade de estudantes universitários (Prado, Kurebayashi \& Silva, 2012) e houve também resultados positivos com a redução de ansiedade e dor em profissionais de enfermagem de um hospital em decorrência do tratatamento com auriculoterapia (Kurebayashi et al., 2017).

$\mathrm{Na}$ literatura internacional, uma pesquisa realizada em um serviço de acupuntura no hospital em Riobamba, Equador, com um número maior de participantes mulheres, diagnosticadas com transtorno de ansiedade, teve como resultado a melhora nas respostas emocionais das mulheres, depois de um período de 12 semanas de tratamento com a auriculopuntura, sendo esta prática, uma alternativa de tratamento para estresse e ansiedade (Barceló, Goyes, López \& Leyva, 2018).

Sobre a utilização da acupuntura, os secretários mencionaram que ela foi eficaz para a melhora na hérnia de disco, além conhecer pessoas que tiveram respostas positivas ao utilizar a PIC em sequela de acidente vascular cerebral. Estudos também comprovam que a acupuntura melhora de modo significativo, a dor provocada pela hérnia de disco lombar (Rigo et al., 2011; Carvalho et al., 2013). A literatura ainda aponta que a acupuntura é eficaz para o tratamento de dores de cabeça, frequentes, episódicas ou tensionais crônicas (Linde et al., 2016).

Outra PIC mencionada no que se refere a recurso de saúde foi a quiropraxia, o secretário apontou que ela tem resultados positivos para quem tem dores na coluna. Estudos comprovam que as técnicas de manipulação, exemplo da quiropraxia, promoveram o alívio de dor de maneira mais rápida mais prolongada nos pacientes com dor cervical (Silva, Lima, Costa \& Silva, 2018).

A musicoterapia também foi mencionada, quando os secretários foram questionados em relação à eficácia das PIC. A literatura aponta que a música minimizou os desconfortos de mulheres em situação de trabalho de parto e facilitou a adaptação do bebê nos primeiros meses de vida (Tabarro, Campos, Galli, Novo \& Pereira, 2010), corroborando com a ideia do secretário de que a música promove o relaxamento das mulheres nestas condições. A literatura ainda aponta outras PIC eficazes para o trabalho de parto, a exemplo da Auriculoterapia e Hidroterapia, que colaboram para a redução da dor e da ansiedade (Silva, Cunha \& Araújo, 2020). 
Os secretários também citaram a homeopatia enquanto recurso de saúde utilizada por eles, mas eles mencionaram não acreditar na eficácia desta prática, uma vez esta PIC se dá pela diluição da medicação em várias proporções, e outro secretário mencionou que era esperado uma potência da medicação a curto prazo, o que não aconteceu. Ressalta-se que um dos secretários viu a eficácia da homeopatia ao ser utilizada por um conhecido, mencionando que a crença dela não a mesma que a sua.

A desconfiança da eficácia da homeopatia também foi verificada em um estudo com gestores, onde não há compreensão da ação das ultradiluições pra fazer a medicação homeopática, desta maneira, as ultradiluições são apontadas como o ponto principal da resistência em acreditar na eficácia desta PIC (Salles \& Schraiber, 2009).

Sobre a temática da formação acadêmica envolvendo as PIC, apenas 5 dos secretários entrevistados disseram que tiveram contato com a temática das PIC. A formação profissional sem conteúdo de PIC também foi abordada no estudo com profissionais que trabalhavam em uma UBS, sendo que 68,2\% não tiveram nada relacionado ao tema, 24,6\% afirmaram ter tido algum conteúdo e 7,2\% não se recordavam (Carvalho \& Nóbrega, 2017). No tocante a terem recebido alguma capacitação/curso que abordassem as PIC, 23,2\% responderam afirmativamente, 76,8\% negam qualquer preparo após a inserção no trabalho. (Carvalho \& Nóbrega, 2017).

Uma pesquisa realizada por Salles, Homo e Silva (2014) através da busca de dados por meio dos sites de 209 instituições públicas de ensino superior de Enfermagem, Medicina e Fisioterapia no Brasil, entre os anos de 2012 a 2013, identificou que somente 43 (32,3\%) oferecem disciplinas relacionadas com o tema. Dentre os três cursos, as escolas de Enfermagem oferecem mais disciplinas (26,4\%), seguidas pela Medicina (26,4\%) e Fisioterapia (14,6\%), sendo que a maioria delas são oferecidas de forma optativa. Destaca-se ainda que no Brasil há pouca informação organizada que contemple a oferta de ensino em PIC, seja na graduação ou pós-graduação (Nascimento et al., 2018).

Um dos entraves que pode dificultar a implantação das PIC na RAS é a falta de formação nas universidades para os profissionais da saúde com esta temática. Esta realidade foi identificada entre os gestores, em que apenas 5 deles estudaram as PIC durante a formação acadêmica. Mas esta realidade ainda prevalece no Brasil, onde há uma lacuna na grade curricular dos cursos de saúde que contemple as PIC. Os dados expostos acima reforçam a necessidade da reformulação nas grades curriculares dos cursos na área da saúde, considerando a pouca abordagem da Política Nacional e as PIC na formação dos estudantes desta área (Fischborn et al., 2016) de modo a possibilitar aos estudantes e futuros profissionais do campo da saúde, a compreensão de novas práticas no seu contexto de atuação e que estejam de acordo à lógica de cuidado na integralidade em saúde e à promoção global do cuidado humano.

\section{Considerações Finais}

O estudo permitiu mostrar a compreensão dos secretários de saúde do NRS-Sul sobre as PIC e aspectos desta temática. Identificou-se que os secretários conhecem várias PIC e já utilizaram algumas delas para recursos terapêuticos, obtendo resultados positivos. Entretanto, a PNPIC só é conhecida por uma minoria, sendo que o desconhecimento da política pode oferecer entraves para a implantação das PIC nos diversos serviços da Rede de Atenção à Saúde.

Há ainda uma lacuna na formação acadêmica dos secretários em relação à temática das PIC, sendo esta a realidade dos profissionais de saúde do país, o que revela a importância das universidades incluírem na formação dos estudantes disciplinas referentes às práticas integrativas, valorizando desta forma, que os profissionais compreendam outros recursos terapêuticos além das ações da medicina convencional.

Destaca-se também a necessidade de articulação entre os diversos atores, como os profissionais, usuários, instituições, gestores de saúde, comunidade a fim de garantir o fortalecimento das PIC enquanto recurso terapêutico potente para ações de 
promoção da saúde e reconhecimento dos saberes tradicionais e populares nos diversos modos de pensar a saúde e os variados modos de adoecimento.

\section{Referências}

Albear de la Torre, D., Cedeño R. E., Fajardo, C. S., \& Riveaux, V. R. (2016 ). Eficacia de la auriculoterapia en pacientes con sacrolumbalgia aguda. MEDISAN, Santiago de Cuba, 20(6): 739-45.

Barceló, N. M., Goyes, A. R. O., López, I. L. M., \& Leyva, B. G. (2018). Impacto social de la auriculoterapia en pacientes con estrés y ansiedad. Chakiñan, Revista de Ciencias Sociales y Humanidades, [S.1.](5): 146-156.

Bardin, L. (2011). Análise de Conteúdo: Edições 70.

Carvalho, J. L. S., \& Nobrega, M. P. S. S. (2017). Práticas integrativas e complementares como recurso de saúde mental na Atenção Básica. Rev. Gaúcha Enferm., Porto Alegre, 38(4): e2017-0014.

Carvalho, L. B., Oyakawa, A., Martins, R. S., Castro, P. C. G., Ferreira, L. M. N., Melo, J. S. A., et al. (2013). Hérnia de disco lombar: tratamento. Acta Fisiatr, 20(2): 75-2.

Contatore, O. A. Barros, N. F., Durval, M. R. Barrio, P. C. C. C., Coutinho, B. D., Santos, J. A. et al. (2015). Uso, cuidado e política das práticas integrativas e complementares na Atenção Primária à Saúde. Ciênc. saúde coletiva, 20(10): 3263-73.

Fertonani, H. P., Pires, D. E. P., Biff, D., \& Scherer, M. D. A. (2015). Modelo assistencial em saúde: conceitos e desafios para a atenção básica brasileira. Ciênc. saúde coletiva, Rio de Janeiro, 20(6): 1869-78.

Fischborn, A. F., Machado, J., Fagundes, N. C., \& Pereira, N. M. A Política das Práticas Integrativas e Complementares do SUS: o relato da implementação em uma unidade de ensino e serviço de saúde. Cinergis, Santa Cruz do Sul, 17(4): 358-63.

Fontanella, B. J. B., Ricas, J., \& Turato, E. R. (2008). Amostragem por saturação em pesquisas qualitativas em saúde: contribuições teóricas. Cad. Saúde Pública, Rio de Janeiro, 24(1): 17-27.

Galhardi, W. M. P., Barros, N. F., \& Leite-Mor, A. C. M. B. (2013). O conhecimento de gestores municipais de saúde sobre a Política Nacional de Prática Integrativa e Complementar e sua influência para a oferta de homeopatia no Sistema Único de Saúde local. Ciênc. saúde coletiva, Rio de Janeiro, 18(1): 21320 .

Ministério da Saúde. Secretaria de Atenção à Saúde. Departamento de Atenção Básica. Política Nacional de Práticas Integrativas e Complementares no SUS : atitude de ampliação de acesso / Ministério da Saúde. Secretaria de Atenção à Saúde. Departamento de Atenção Básica - (2ª ed.) - Brasília, DF.

Santos, A. M., \& Giovanella, L. (2014). Governança regional: estratégias e disputas para gestão em saúde. Rev Saúde Pública, 48(4): 622-31.

Instituto Brasileiro de Geografia e Estatística,. https://cidades.ibge.gov.br/.

Ischkanian, P. C., \& Pelicioni, M. C. F. (2012). Desafios das práticas integrativas e complementares no sus visando a promoção da saúde. Rev. bras. crescimento desenvolv. hum., São Paulo, 22(1): 233-8.

Kurebayashi, L. F. S., Turrini, R. N. T., Souza, T. P. B., Marques, C. F., Rodrigues, R. T. F., \& Charlesworth, K. (2017). Auriculoterapia para redução de ansiedade e dor em profissionais de enfermagem: ensaio clínico randomizado. Rev. Latino-Am. Enfermagem, Ribeirão Preto, 25 : e2843.

Linde, K., Allais, G., Brinkhaus, B., Fei, Y., Mehrin, G., Shin, B. C., Vickers, A., \& Whit, A. R. (2016). Acupuncture for the prevention of tension-type headache. Cochrane Database of Systematic Reviews, Issue 4. Art .n:CD007587.

Luz, M. T. (2005). Cultura Contemporânea e Medicinas Alternativas: Novos Paradigmas em Saúde no Fim do Século XX. Physis: Rev. Saúde Coletiva, 15 (Suplemento): 145- 76 .

Melchart, D. (2018). From Complementary to Integrative Medicine and Health: Do We Need a Change in Nomenclature? Complement Med Res, 25: 76-8.

Minayo, M. C. S. (2009). O desafio da pesquisa social. In: Minayo, M.C.S.; Deslandes, S.F. \& Gomes, R. Pesquisa Social. Teoria, método e criatividade. (28a ed.), Editora Vozes, p. 9-30.

Nascimento, M. C., Barros, N. F., Nogueira, M. I., \& Luz, M. T. (2013). A categoria racionalidade médica e uma nova epistemologia em saúde. Ciênc. saúde coletiva, Rio de Janeiro, 18(12): 3595-604.

Nascimento, M. C., Romano, V. F., Chazan, A. C. S., \& Quaresma, C. H. (2018). Formação em práticas integrativas e complementares em saúde: desafios para as universidades públicas. Trab. educ. saúde, Rio de Janeiro, 16 (2); 751-772.

Prado, J. M., Kurebayashi, L. F. S., \& Silva, M. J. P. (2012). Eficácia da auriculoterapia na redução de ansiedade em estudantes de enfermagem. Rev. esc. enferm. USP, São Paulo, 46( 5): 1200-1206.

Rigo, J. C., Pires, A. P. M., Oliveira, L. A., Harres, S. S., \& Dalacorte, R. R. (2011). Evidências da acupuntura no tratamento da dor lombar. Geriatr Gerontol Aging, 5(3): 175-179.

Sacramento, H. T. (2017). Vitória (ES): experiência exitosa em práticas integrativas e complementares. J Manag Prim Heal Care, .8(2): 333-342, 
Research, Society and Development, v. 10, n. 5, e30510514905, 2021

(CC BY 4.0) | ISSN 2525-3409 | DOI: http://dx.doi.org/10.33448/rsd-v10i5.14905

Salles, L. F., Bel Homo, R. F., \& Silva, M. J. P. (2014). Situação do ensino das práticas integrativas e complementares nos cursos de graduação em enfermagem, fisioterapia e medicina. Cogitare Enfermagem, 19(4):741-6.

Salles, S. A. C., \& Schraiber, L. B. (2009). Gestores do SUS: apoio e resistências à Homeopatia. Cad. Saúde Pública, Rio de Janeiro, 25(1): 195-202.

Santos, F. A. S., Sousa, I. M. C., Gurgel, I. G. D., Bezerra, A. F. B., \& Barros, N. F. (2011). Política de práticas integrativas em Recife: análise da participação dos atores. Rev. Saúde Pública, São Paulo, 45(6): 1154-59.

Silva, A. D. V., Cunha, E. A., \& Araújo, R. V. (2020). The benefits of integrative and complementary practices in childbirth work. Research, Society and Development, 9(7): 1-16, e614974468.

Silva, R. M. V., Lima, M. S., Costa, F. H., \& Silva, A. C. (2018). Efeitos da quiropraxia em pacientes com cervicalgia: revisão sistemática. Rev. dor, São Paulo, 13(1): 71-4.

Tabarro, C. S., Campos, L. B., Galli, N. O., Novo, N. F., \& Pereira, V. M. (2010). Efeito da música no trabalho de parto e no recém-nascido. Rev Esc Enferm USP, 44(2): 445-52.

World Health Organization .Who. (2013). Who Traditional Medicine Strategy 2014-2023: World Health Organization. 